\title{
$A B C G 2$ rs2231142 variant in hyperuricemia is modified by SLC2A9 and SLC22A12 polymorphisms and cardiovascular risk factors in an elderly community-dwelling population
}

\author{
Jia Liu', Wei Yang ${ }^{1 *}$ (id, Yun Li', Zhanyun Wei ${ }^{1}$ and Xiaojuan Dan²
}

\begin{abstract}
Background: The ABCG2 rs2231142 single nucleotide polymorphism (SNP) is one of the most significant genetic variants associated with hyperuricemia (HUA) in Asian populations. However, the risk of $A B C G 2$ rs2231142 variants for HUA could interact with other important HUA risk variants and cardiovascular factors. This study investigated the effects of the combined association among ABCG2 rs2231142 and multiple HUA genetic variants or cardiovascular risk factors on HUA risk and serum uric acid (sUA) levels in an elderly Chinese population.

Methods: A total of 1206 participants over 65 years old were enrolled in this study. Physical and laboratory examinations were performed for all participants. The ABCG2 rs2231142, SLC2A9 rs3733591, and SLC22A12 rs893006 SNPs were assayed using a standardized protocol. Logistic regression analysis and liner regression were adjusted respectively to account for the association between ABCG2 rs2231142 and other genetic variants, as well as between cardiovascular risk factors and HUA risk and sUA levels.

Results: The prevalence of HUA was $14.71 \%$ in the elderly community-dwelling population. The ABCG2 rs 2231142 risk T allele was associated with HUA risk (odds ratio $(\mathrm{OR})=1.63,95 \%$ confidence interval $(\mathrm{Cl})$ : 1.27-2.11; $p=1.65 \times$ $10^{-4}$ ) and with increased sUA levels (Beta $=0.16, p=6.75 \times 10^{-9}$ ) in the whole study population. Linear regression analysis showed that the mean sUA level increased linearly with the number of risk alleles of the three candidate genetic variants (Beta $=0.18, p=1.94 \times 10^{-12}$ ) The joint effect of the ABCG2 rs2231142 T allele and cardiovascular risk factors (obesity, hypertension and dyslipidemia) was also associated with increased HUA risk and sUA levels. Each copy of the risk T allele was significantly associated with enhanced HUA risk in patients with hypertriglyceridemia ( $\mathrm{OR}=2.52,95 \% \mathrm{Cl}: 1.33-4.60 ; p=0.003)$ compared to controls.

Conclusion: Our findings reinforce the importance of the ABCG2 rs2231143 variant as a crucial genetic locus for HUA in Chinese populations and demonstrated the combined effects of multiple genetic risk variants and cardiovascular risk exposures on HUA risk and increased sUA level.
\end{abstract}

Keywords: Uric acid, $A B C G 2$, Polymorphisms, Hypertension, Triglyceridemia

\footnotetext{
*Correspondence: yangw_79@163.com

'Departments of Geriatric Medicine, Xuanwu Hospital, The Capital Medical University, 45 Changchun Street, Xicheng District, Beijing 100053, China

Full list of author information is available at the end of the article
}

(c) The Author(s). 2020 Open Access This article is licensed under a Creative Commons Attribution 4.0 International License, which permits use, sharing, adaptation, distribution and reproduction in any medium or format, as long as you give appropriate credit to the original author(s) and the source, provide a link to the Creative Commons licence, and indicate if changes were made. The images or other third party material in this article are included in the article's Creative Commons licence, unless indicated otherwise in a credit line to the material. If material is not included in the article's Creative Commons licence and your intended use is not permitted by statutory regulation or exceeds the permitted use, you will need to obtain permission directly from the copyright holder. To view a copy of this licence, visit http://creativecommons.org/licenses/by/4.0/. The Creative Commons Public Domain Dedication waiver (http://creativecommons.org/publicdomain/zero/1.0/) applies to the data made available in this article, unless otherwise stated in a credit line to the data. 


\section{Background}

Hyperuricemia (HUA) is defined by high serum uric acid (sUA) levels due to an imbalance of purines from food, urate synthesis, and/or urate excretion into the urine or gastrointestinal tract [1], which is related to gout, diabetes, hypertension, cardiovascular disease, and kidney disease [1-3]. The cause of HUA is multi-factorial, including age, gender, obesity, diet, alcohol consumption, insulin resistance, hypertension, and medication [1, 4]. sUA, however, is under strong genetic control, and more than ten sUA associated genes have been identified in genome-wide association studies (GWAS) [5, 6], including three main risk genes: ATP-binding cassette subfamily G member 2 (ABCG2), glucose transporter type 9 (GLUT9, also known as SLC2A9), and urate anion transporter 1 (URAT1, also known as $S L C 22 A 12$ ).

$A B C G 2$ encodes a high-capacity urate efflux transporter $[5,7]$. A single nucleotide polymorphism (SNP) rs2231142 (Q141K) located in exon 5 of $A B C G 2$ leads to a Glu141Lys amino acid substitution [7, 8]. The $\mathrm{T}$ allele of $\mathrm{rs} 2231142$ has been associated with increased serum urate levels, and its frequency is approximately 3-fold higher in East Asian populations compared to European populations [6, 9]. The $A B C G 2$ rs2231142 SNP is reported to be the most significant genetic variant associated with HUA and gout in East Asia compared to other sUA-risk genes [6,9]. However, the association between HUA and the $A B C G 2$ variants interacting with $S L C 2 A 9$ and SLC22A12 has not been investigated in a Chinese population. In addition, few studies have identified an association of lifestyle-related risk factors (smoking, alcohol consumption, Aspirin use) or disease conditions (hypertension, obesity) with ABCG2 rs2231142related HUA risk. Therefore, in this study, we determined if SNPs of three major sUA genes (ABCG2 rs2231142, SLC2A9 rs3733591, and SLC22A12 rs893006), individually and/or in combination were associated with HUA risk and sUA level. We also investigated the association between HUA and cardiovascular risk factors within the $A B C G 2$ risk allele carriers, to better understand interactions of genecardiovascular risk factors and to develop personalized treatment strategies for $A B C G 2$ rs2231142-related HUA.

\section{Methods}

\section{Ethics statement}

This study was approved by the Ethics Committee of Xuanwuu Hospital (Beijing, China) and all participants signed informed consent before enrolling into this study.

\section{Study design and subjects}

Subjects were recruited from the Beijing Communitydwelling Study on Aging cohort between 2015 and 2016. The study was designed to explore the health status in older adults. Using a stratified multiphase sampling design, a total of 1260 individuals who included representative samples from the urban district in Beijing were enrolled. Subjects with missing sUA data $(n=5)$ or DNA sequencing data $(n=30)$, as well as history of severe chronic kidney disease [endogenous creatinine clearance $(\mathrm{Ccr})<30 \mathrm{ml} / \mathrm{min}$ ] $(n=19)$ were excluded. To ensure that estrogen did not influence sUA levels in females, we recruited both males $(n=$ $699)$ and females $(n=507)$ who were $\geq 65$ years old. HUA was defined as a serum urate acid level $>417 \mu \mathrm{mol} / \mathrm{L}(7.0$ $\mathrm{mg} / \mathrm{dl})$ in males and $>357 \mu \mathrm{mol} / \mathrm{L}(6.0 \mathrm{mg} / \mathrm{dl})$ in females according to a previous study [10]. The criteria for the controls included individuals without history and/or current treatment of HUA or gout, and sUA levels of $\leq 417$ (7.0 mg/ $\mathrm{dl})$ in males and $\leq 357 \mu \mathrm{mol} / \mathrm{L}(6.0 \mathrm{mg} / \mathrm{dl})$ in females.

\section{Collection of laboratory test and physical examination data}

Venous blood was collected from each subject in the morning after fasting for at least $12 \mathrm{~h}$. Samples were analyzed for fasting blood glucose (FBG), triglyceride (TG), low-density lipoprotein (LDL), high-density lipoprotein (HDL) levels, and sUA using IPE (www.ipe-bio.com) in Beijing. sUA levels were assessed using uricase/peroxidase methods. Resting blood pressure (BP) was measured by well-trained personnel using the auscultatory method and the phase I and V (sudden reduction/disappearance) Korotkoff sounds to identify systolic BP (SBP) and diastolic BP (DBP), respectively. Each participant was seated comfortably in a quite environment for $5 \mathrm{~min}$ before BP measurements. BP levels were measured in both upper arms at the first office visit to detect possible difference between arms, and the arm with the higher BP value was used for subsequent measurements with an appropriate cuff size for the arm circumference. Three BP measurements were recorded within 1-2 min of each measurement and the average of the three measurements was used for data analysis. The office hypertension was defined as office SBP $\geq 140 \mathrm{mmHg}$ and/or DBP $\geq 90 \mathrm{mmHg}$ in any individual, and subjects who had been receiving antihypertensive medication were also defined as hypertensive [11]. Diabetes mellitus was diagnosed based on the guidelines of the American Diabetes Association (ADA). Body mass index (BMI) was calculated as weight (kg) / height $\left(\mathrm{m}^{2}\right)$ and obesity was defined as BMI $\geq 24$ $\mathrm{kg} / \mathrm{m}^{2}$. High levels of TG and LDL were defined as $\geq 1.7$ $\mathrm{mmol} / \mathrm{L}$ and $\geq 3.4 \mathrm{mmol} / \mathrm{L}$, respectively, and low HDL levels were defined as $<1.0 \mathrm{mmol} / \mathrm{L}$ according to a previous study [12]. Other lifestyle information, such as tobacco smoking and alcohol consumption, was recorded through face-to-face questionnaires.

\section{SNP selection and genotyping}

Three SNPs, including ABCG2 rs2231142, SLC2A9 rs3733591, and SLC22A12 rs893006, were selected based on previously reported associations with sUA levels, 
especially in Asian populations [8,13-15]. The minor allele frequencies (MAF) of these selected SNPs were $\geq 5 \%$ in Chinese populations according to the 1000 Genomes Projects (http://www.internationalgenome.org/) database.

Genomic DNA was extracted from peripheral blood samples of each participant using a standard procedure, genotyped for the three SNPs using the TaqMan assay (Applied Biosystems, Foster City, CA), and then analyzed for allelic discrimination using the ABI PRISM 7900 Sequence Detection System and SDS software (Applied Biosystems).

\section{Statistical analysis}

Continuous variables are summarized as mean \pm standard deviation (SD) and were analyzed using Student's $t$ test for normal data distributions or the Mann-Whitney $U$ test for non-normal data distributions. Comparisons of categorical variables were analyzed using the Chisquare test. The association of risk allele frequencies of these three loci with HUA was analyzed based on gender. The association between the risk allele of three loci and sUA level or HUA was analyzed using the linear regression model and logistical model, respectively, with justification for age, gender, hypertension, serum creatinine, BMI, TG, HDL, and LDL. The association of three SNP genotypes and sUA levels was analyzed using the one-way ANOVA with a posthoc Bonferroni test and a Bonterroni correction of $p<0.0167$ (equivalent to $p<0.05$ significance). The genetic risk score of individuals was generated by counting the number of risk alleles of high sUA levels in the study population, ranging from 0 to 6 (C allele of SLC2A9 rs3733951, T allele of ABCG2 rs2231142, and G allele of SLC22A12 rs893006). The association between genetic risk score and sUA levels was analyzed using a linear regression model after adjusting for the above listed covariates. To assess the effects of cardiovascular risk factors on the risk of HUA associated with the ABCG2 $\mathrm{T}$ allele, multiple logistic regression analysis was also conducted. Odds ratios (OR) and $95 \%$ confidence intervals $(95 \% \mathrm{CI})$ were then calculated. All data were analyzed using SPSS version 17.0 (SPSS, Chicago, IL, USA), and a $p$ value $<0.05$ was considered statistically significant.

\section{Results}

\section{Characteristics of study population}

A total of 1026 individuals, including 699 males and 507 females, were enrolled in this study. The average age of the participants was $76.64 \pm 6.40$ years (range, 65-94 years) and all were from an elderly Beijing communitydwelling population. The prevalence of HUA was $14.71 \%$ in the study population, with 151 HUA cases and 1055 controls. Table 1 indicates that individuals in the HUA group had higher levels of serum creatinine, TG, LDL, and BMI, but lower levels of HDL, compared
Table 1 Basic clinical and biochemical characteristics of participants

\begin{tabular}{llll}
\hline Variables & $\begin{array}{l}\text { Hyperuricemia } \\
n=151\end{array}$ & $\begin{array}{l}\text { Control } \\
n=1055\end{array}$ & $p$-value \\
\hline sUA ( $\mathrm{mmmol} / \mathrm{L})$ & $441.74 \pm 53.53$ & $290.31 \pm 60.81$ & - \\
Age & $77.01 \pm 6.90$ & $76.59 \pm 6.32$ & 0.66 \\
Male (n, \%) & $78(51.7)$ & $621(58.9)$ & 0.095 \\
TG (mmol/L) & $2.07 \pm 1.26$ & $1.43 \pm 0.84$ & $8.10 \times 10^{-14}$ \\
LDL (mmol/L) & $3.47 \pm 0.78$ & $3.22 \pm 0.81$ & 0.001 \\
HDL(mmol/L) & $1.24 \pm 0.30$ & $1.35 \pm 0.32$ & $2.22 \times 10^{-5}$ \\
FBG (mmol/L) & $6.01 \pm 1.50$ & $6.05 \pm 1.88$ & 0.31 \\
Serum creatinine ( $\mu$ mol/L) & $72.25 \pm 12.24$ & $68.83 \pm 13.19$ & 0.003 \\
BMI (kg/m $\left.{ }^{2}\right)$ & $26.85 \pm 2.34$ & $24.14 \pm 2.98$ & $1.01 \times 10^{-26}$ \\
Current drinking (\%) & $30(19.9)$ & $248(23.5)$ & 0.35 \\
Current smoking (\%) & $34(22.5)$ & $255(24.2)$ & 0.68 \\
Office SBP (mmHg) & $129.34 \pm 15.65$ & $127.71 \pm 11.61$ & 0.84 \\
Office DBP (mmHg) & $78.54 \pm 8.66$ & $79.20 \pm 11.69$ & 0.25 \\
Hypertension (\%) & $72(47.7)$ & $409(38.8)$ & 0.041 \\
Hypertension treatment & $48(31.8)$ & $198(18.8)$ & $4.90 \times 10^{-5}$ \\
Diuretic (\%) & $6(4.0)$ & $42(4.0)$ & 0.99 \\
Diabetes (\%) & $30(19.9)$ & $192(18.2)$ & 0.65 \\
\hline
\end{tabular}

to controls $(p<0.05)$. The percentage of hypertensive patients in the HUA cases was also higher compared to the control group $(47.7 \%$ vs $38.8 \%, p=0.041)$, but there was no significant difference in SBP and DBP between the two groups, which may be related to hypertension treatment (e.g., $31.8 \%$ of the HUA cases and $18.8 \%$ of the controls, $p=4.9 \times 10^{-5}$ ). However, the ratio of diuretics was relatively low and did not reach statistical significance between the two groups. Moreover, there was no significant difference in FBG, alcohol consumption, tobacco smoking, or history of diabetes between the case and control groups.

\section{Distribution of $A B C G 2, S L C 2 A 9$, and SLC22A12 polymorphisms between two groups}

The three tested SNPs (SLC2A9 rs3733591, ABCG2 rs2231142, and $S L C 22 A 12$ rs893006) were in HardyWeinberg equilibrium $(p=0.30,0.70$, and 0.33 , respectively). The genotype and allele frequencies of these three SNPs are shown in Table 2 and stratified by gender. In the general population, the genotype frequencies of ABCG2 rs2231142 among HUA cases were $11.9 \%(\mathrm{~T} / \mathrm{T})$, $53.6 \%(\mathrm{~T} / \mathrm{G})$, and $34.4 \%(\mathrm{G} / \mathrm{G})$, respectively, while the genotype frequencies were $8.0 \%(\mathrm{~T} / \mathrm{T}), 40.4 \%(\mathrm{~T} / \mathrm{G})$, and $51.7 \%(\mathrm{G} / \mathrm{G})$ in the controls, respectively. The minor $\mathrm{T}$ allele of the $A B C G 2$ variants reached $28.2 \%$ in the controls and $38.7 \%$ in the HUA cases. The distribution of $A B C G 2$ variants showed statistical significance in males and when combining males and females for the Chi- 
Table 2 Distribution of three SNPs in HUA cases and controls, stratified by gender

\begin{tabular}{|c|c|c|c|c|c|c|}
\hline \multirow{2}{*}{$\begin{array}{l}\text { Gene/ } \\
\text { SNP }\end{array}$} & \multicolumn{2}{|l|}{ Male } & \multicolumn{2}{|l|}{ Female } & \multicolumn{2}{|l|}{ Total } \\
\hline & $\begin{array}{l}\text { Case (\%) } \\
(n=78)\end{array}$ & $\begin{array}{l}\text { Control (\%) } \\
(n=621)\end{array}$ & $\begin{array}{l}\text { Case (\%) } \\
(n=73)\end{array}$ & $\begin{array}{l}\text { Control (\%) } \\
(n=434)\end{array}$ & $\begin{array}{l}\text { Case (\%) } \\
(n=151)\end{array}$ & $\begin{array}{l}\text { Control (\%) } \\
(n=1055)\end{array}$ \\
\hline \multicolumn{7}{|c|}{ SLC2A9 rs3733591 } \\
\hline $\mathrm{C} / \mathrm{C}$ & $10(12.8)$ & $72(11.6)$ & $13(17.8)$ & $33(7.6)$ & $23(15.2)$ & $105(10.0)$ \\
\hline $\mathrm{C} / \mathrm{T}$ & $34(43.6)$ & $284(45.7)$ & $38(52.1)$ & $198(45.6)$ & $72(47.4)$ & $482(45.7)$ \\
\hline$T / T$ & $34(43.6)$ & $265(42.7)$ & $22(30.1)$ & $203(46.8)$ & $56(37.1)$ & $468(44.4)$ \\
\hline$p^{\mathrm{a}}$ & 0.91 & & 0.003 & & 0.075 & \\
\hline \multicolumn{7}{|c|}{ Allele (\%) } \\
\hline C & $54(34.6)$ & $428(34.5)$ & $604(69.6)$ & $264(30.4)$ & $118(39.1)$ & $692(32.8)$ \\
\hline T & $102(65.4)$ & $814(65.5)$ & $82(56.2)$ & $64(43.8)$ & $184(60.9)$ & $1418(67.2)$ \\
\hline \multicolumn{7}{|c|}{ ABCG2 rs2231142 } \\
\hline$T / T$ & $8(10.3)$ & $50(8.1)$ & $10(13.7)$ & $34(7.8)$ & $18(11.9)$ & $84(8.0)$ \\
\hline $\mathrm{T} / \mathrm{G}$ & $46(59.0)$ & $253(40.7)$ & $35(47.9)$ & $173(39.9)$ & $81(53.6)$ & $426(40.4)$ \\
\hline $\mathrm{G} / \mathrm{G}$ & $24(30.8)$ & $318(51.2)$ & $28(38.4)$ & $227(52.3)$ & $52(34.4)$ & $545(51.7)$ \\
\hline$p^{a}$ & 0.003 & & 0.053 & & $6.37 \times 10^{-5}$ & \\
\hline \multicolumn{7}{|c|}{ Allele (\%) } \\
\hline T & $62(39.7)$ & $353(28.4)$ & $55(37.7)$ & $241(27.8)$ & $117(38.7)$ & $594(28.2)$ \\
\hline G & $94(60.3)$ & $889(71.6)$ & $91(62.3)$ & $627(72.2)$ & $185(61.3)$ & $1516(71.8)$ \\
\hline \multicolumn{7}{|c|}{ SLC22A12 rs893006 } \\
\hline $\mathrm{G} / \mathrm{G}$ & $51(65.4)$ & $346(55.7)$ & $39(53.4)$ & $218(50.2)$ & $90(59.6)$ & $564(53.5)$ \\
\hline $\mathrm{G} / \mathrm{T}$ & $25(32.1)$ & $234(37.7)$ & $31(42.5)$ & $187(43.1)$ & $56(37.1)$ & $421(39.9)$ \\
\hline $\mathrm{T} / \mathrm{T}$ & $2(2.6)$ & 419 (6.6) & $3(4.1)$ & $29(6.7)$ & $5(3.3)$ & $70(6.6)$ \\
\hline$p^{\mathrm{a}}$ & 0.16 & & 0.67 & & 0.17 & \\
\hline \multicolumn{7}{|c|}{ Allele (\%) } \\
\hline G & $127(81.4)$ & $926(74.6)$ & $109(74.7)$ & $623(71.8)$ & $561(26.6)$ & $1549(73.4)$ \\
\hline $\mathrm{T}$ & 29 (18.6) & $316(25.4)$ & $37(25.3)$ & $245(28.2)$ & 66 (21.9) & $236(78.1)$ \\
\hline
\end{tabular}

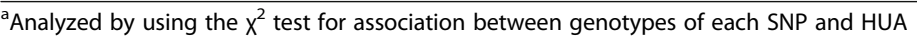

square test, there was no significant association in females $(p=0.053)$. The distribution of $S L C 2 A 9$ rs 3733591 was only significantly different in females $(p=0.003)$, with a frequency of $17.8 \%$ for $\mathrm{C} / \mathrm{C}$ and $52.1 \%$ for $\mathrm{C} / \mathrm{T}$ in the HUA cases. There was no significant difference in genotype frequency of SLC22A12 rs893006 between HUA cases and controls in both males and females.

\section{Association between risk alleles of uric acid genes with sUA levels and HUA risk}

In all samples, there was a significant association of increased sUA levels $(\mu \mathrm{mol} / \mathrm{L})$ with each copy of risk allele of the three SNPs (Beta $=0.08, p=0.002$ in rs3733591 SLC2A9, Beta $=0.16, p=6.75 \times 10^{-9}$ in rs2231142 ABCG2, Beta $=0.07, p=0.006$ in rs893006 SLC22A12; Table 3). There was also a significant association HUA with the risk alleles of these three SNPs [OR (95\% CI): 1.34 (1.03-1.74), $p=0.029$ in rs3733591 SLC2A9, OR (95\% CI): 1.63 (1.27$2.11), p=1.65 \times 10^{-4}$ in rs2231142 ABCG2]; however, there was no significant association of HUA with SLC22A12 rs893006 variants [OR (95\% CI): 1.33 (0.99-1.78), $p=$ 0.061]. After we separated the subjects by gender, the $\mathrm{T}$ allele of rs2231142 $A B C G 2$ was associated with sUA level and HUA in both males and females (all $p<0.05$ ), but the $C$ risk allele in rs3722591 SLC2A9 was only associated with sUA level and HUA in females. The SLC22A12 rs893006 G risk allele was only associated with sUA level in males.

\section{Association between different genotypes of three SNPs and sUA level}

sUA level $(\mu \mathrm{mol} / \mathrm{L})$ was associated with the $A B C G 2$ rs2231142 polymorphism in all populations (T/T: $337.46 \pm$ 71.25, T/G: $317.51 \pm 80.53, \mathrm{G} / \mathrm{G}: 287.46 \pm 75.13 ; p=6.98 \times$ $10^{-8}$ ) and sUA levels of the G/G genotypes were significantly lower compared with those of the $\mathrm{T} / \mathrm{T}$ genotypes $\left(p=4.40 \times 10^{-6}\right)$. There was also a significant difference in sUA levels in the G/G and T/T male carriers (G/G vs. T/T: $313.32 \pm 73.83$ vs $348.20 \pm 68.85 ; p=0.003)$ and female carriers (G/G vs T/T: $276.18 \pm 71.65$ vs $323.32 \pm 72.67 ; p=$ $\left.2.67 \times 10^{-4}\right)$. The sUA level was higher in SLC2A9 C/C 
Table 3 Association of risk alleles of uric acid genes with sUA levels and HUA

\begin{tabular}{|c|c|c|c|c|c|c|c|}
\hline \multirow[t]{2}{*}{ Gene } & \multirow[t]{2}{*}{ SNP } & \multirow{2}{*}{$\begin{array}{l}\text { Coded/ } \\
\text { other } \\
\text { allele }\end{array}$} & \multirow{2}{*}{$\begin{array}{l}\text { CAF } \\
(\%)\end{array}$} & \multicolumn{2}{|l|}{ sUA level } & \multicolumn{2}{|l|}{ Hyperuricemia } \\
\hline & & & & Beta (SE) & $p^{a}$ & OR $(95 \% \mathrm{Cl})$ & $p^{b}$ \\
\hline \multicolumn{8}{|l|}{ Total } \\
\hline SLC2A9 & rs3733591 & $C / T$ & 33.58 & $0.08(3.02)$ & 0.002 & $1.34(1.03-1.74)$ & 0.029 \\
\hline$A B C G 2$ & rs2231142 & $T / G$ & 29.48 & $0.16(3.98)$ & $6.75 \times 10^{-9}$ & $1.63(1.27-2.11)$ & $1.65 \times 10^{-4}$ \\
\hline SLC22A12 & rs893006 & $\mathrm{G} / \mathrm{T}$ & 74.00 & $0.07(3.25)$ & 0.006 & $1.33(0.99-1.78)$ & 0.061 \\
\hline \multicolumn{8}{|l|}{ Male } \\
\hline SLC2A9 & rs3733591 & $C / T$ & 32.35 & $0.05(3.96)$ & 0.122 & $1.09(0.72-1.64)$ & 0.699 \\
\hline$A B C G 2$ & rs2231142 & $T / G$ & 29.69 & $0.16(4.15)$ & $4.48 \times 10^{-7}$ & $1.70(1.20-2.42)$ & 0.003 \\
\hline SLC22A12 & rs893006 & $\mathrm{G} / \mathrm{T}$ & 75.32 & $0.08(4.34)$ & 0.018 & $1.49(0.98-2.28)$ & 0.064 \\
\hline \multicolumn{8}{|l|}{ Female } \\
\hline SLC2A9 & rs3733591 & $C / T$ & 34.48 & $0.13(4.68)$ & 0.002 & $1.89(1.26-2.85)$ & 0.002 \\
\hline$A B C G 2$ & rs2231142 & $T / G$ & 29.19 & $0.17(4.68)$ & $6.56 \times 10^{-5}$ & $1.60(1.10-2.32)$ & 0.013 \\
\hline SLC22A12 & rs893006 & $\mathrm{G} / \mathrm{T}$ & 72.19 & $0.06(4.90)$ & 0.140 & $1.17(0.75-1.84)$ & 0.489 \\
\hline
\end{tabular}

CAF code allele frequency

aLiner regression adjusted by age, gender, BMI, hypertension, serum creatinine, TG, HDL, and LDL

bogistic regression adjusted by age, gender, BMI, hypertension, serum creatinine, TG, HDL, and LDL

carriers of the whole population and females compared with $\mathrm{T} / \mathrm{T}$ carriers $(p=0.005$ and 0.015 , respectively), but there was no association in male subjects. There was also no significant difference in sUA levels between each genotype of the $S L C 22 A 12$ rs893006 polymorphism analyzed using the posthoc Bonferroni test (Table 4).

\section{Association of sUA levels and genetic risk scores}

The genetic risk score of an individual was generated by counting the number of risk alleles of high sUA levels in the study population, ranging from 0 to 6 (C allele of SLC2A9 rs3733951, T allele of ABCG2 rs2231142, and G allele of SLC22A12 rs893006; Fig. 1). Using linear regression analysis, we found that the mean sUA level in both males and females significantly increased with the number of risk alleles (Beta $=0.17, p=6.24 \times 10^{-7}$ in males and Beta $=0.20, p=7.39 \times 10^{-7}$, respectively). In the whole population, the mean sUA level also increased linearly with the number of risk alleles $\left(\right.$ Beta $\left.=0.18, p=1.94 \times 10^{-12}\right)$.

\section{Association between rs2231142 ABCG2 variants and HUA} risk according to cardiovascular risk factors

Table 5 summarizes the association between ABCG2 rs2231142 polymorphisms and HUA risk with or without

Table 4 Association between genotypes of three SNPs and serum urate levels in the study population, separated by gender

\begin{tabular}{|c|c|c|c|c|c|c|c|c|}
\hline \multirow[t]{2}{*}{ Gene (SNP) } & \multirow{2}{*}{$\begin{array}{l}\text { Code /other allele } \\
(1 / 2)\end{array}$} & \multicolumn{3}{|c|}{ SUA (mean $\pm S D) \mu m o l / L$} & \multirow[t]{2}{*}{$p^{a}$} & \multirow{2}{*}{$\begin{array}{l}p^{\mathrm{b}} \\
1 / 1 \text { vs } 1 / 2\end{array}$} & \multirow{2}{*}{$\begin{array}{l}p^{b} \\
1 / 2 \text { vs } 2 / 2\end{array}$} & \multirow{2}{*}{$\begin{array}{l}p^{\mathrm{b}} \\
1 / 1 \text { vs } 2 / 2\end{array}$} \\
\hline & & $1 / 1$ & $1 / 2$ & $2 / 2$ & & & & \\
\hline SLC2A9 rs3733951 & $\mathrm{C} / \mathrm{T}$ & & & & & & & \\
\hline Total & & $327.24 \pm 82.39$ & $311.09 \pm 77.71$ & $302.96 \pm 76.89$ & 0.005 & 0.262 & 0.104 & 0.005 \\
\hline Male & & $337.71 \pm 72.96$ & $326.42 \pm 78.23$ & $324.04 \pm 78.28$ & 0.356 & - & - & - \\
\hline Female & & $308.57 \pm 94.96$ & $290.43 \pm 72.17$ & $274.96 \pm 69.89$ & 0.006 & 0.073 & 0.379 & 0.015 \\
\hline ABCG2 rs2231142 & $\mathrm{T} / \mathrm{G}$ & & & & & & & \\
\hline Total & & $337.46 \pm 71.25$ & $317.51 \pm 80.53$ & $287.46 \pm 75.13$ & $6.98 \times 10^{-8}$ & 0.052 & $5.42 \times 10^{-5}$ & $4.40 \times 10^{-6}$ \\
\hline Male & & $348.20 \pm 68.85$ & $337.90 \pm 78.10$ & $313.32 \pm 73.83$ & $1.85 \times 10^{-5}$ & $1.26 \times 10^{-4}$ & 1.000 & 0.003 \\
\hline Female & & $323.32 \pm 72.67$ & $288.21 \pm 74.89$ & $276.18 \pm 71.65$ & $3.45 \times 10^{-4}$ & 0.012 & 0.236 & $2.67 \times 10^{-4}$ \\
\hline SLC22A12 rs893006 & $\mathrm{G} / \mathrm{T}$ & & & & & & & \\
\hline Total & & $315.33 \pm 80.50$ & $302.80 \pm 75.98$ & $297.60 \pm 66.03$ & 0.012 & 0.023 & 1.000 & 0.186 \\
\hline Male & & $392.11 \pm 79.52$ & $320.66 \pm 72.85$ & $313.54 \pm 63.71$ & 0.087 & - & - & - \\
\hline Female & & $289.41 \pm 75.08$ & $281.58 \pm 74.28$ & $276.17 \pm 64.37$ & 0.402 & - & - & - \\
\hline
\end{tabular}

${ }^{\mathrm{a} A N O V A}$ comparison between groups;

${ }^{\mathrm{b}}$ Bonferroni test; 1 = code allele; 2 = other allele; SNP single-nucleotide polymorphism, SUA serum uric acid 


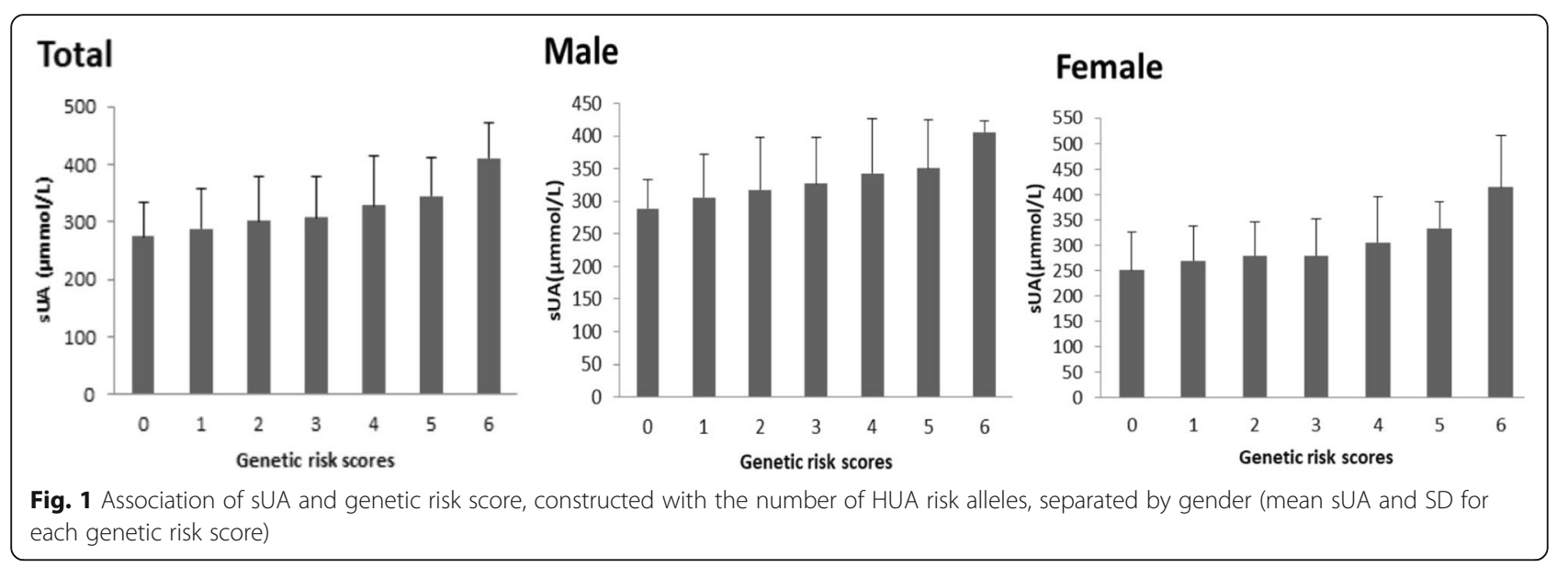

cardiovascular risk factors. In all study populations, the OR of HUA with each copy of the T allele in rs2231142 increased significantly when accounting for cardiovascular risks (such as obesity, hypertension, higher levels of TG and LDL, and lower levels of HDL) compared to those without cardiovascular risk factors. The OR of HUA with high TG levels and low HDL levels had a strong increase in HUA risk associated with each copy of $\mathrm{T}$ alleles in 2,231,142 in males (OR (95\% CI): 2.41 (1.06-4.53), $p=0.035$ and OR (95\% CI): 2.50 (1.12-5.67), $p=0.026$, respectively). Among females, the association was statistically significant in individuals with obesity [OR (95\% CI): 1.59 (1.04-3.04); $p=$ 0.045], hypertension [OR ( $95 \% \mathrm{CI}): 1.83(1.09-3.08) ; p=$ 0.021], and high TG levels [OR (95\% CI): 2.57 (1.10-6.01); $p=0.030$ ] and LDL levels [OR (95\% CI): $1.98(1.20-3.34)$; $p=0.008]$ compared with those with non-cardiovascular risk factors, but not those with low HDL levels ( $p=0.156)$.

We further assessed the association between cardiovascular risk factors and HUA risk or sUA levels among the ABCG2 rs2231142 $\mathrm{T}$ allele carriers (Table 6). BMI and TG levels were associated with higher sUA levels (Beta $=0.21, p=3.40 \times 10^{-8}$ and Beta $=0.24, p=6.18 \times$ $10^{-9}$, respectively) in the whole population, and this association was also significant in males (Beta $=0.23, p=$ $1.14 \times 10^{-5}$ for BMI and Beta $=0.25, p=8.95 \times 10^{-6}$ for TG level, respectively) and females (Beta $=0.20, p=$ 0.002 for BMI and Beta $=0.24, p=4.00 \times 10^{-4}$ for TG level, respectively). The association of BMI and TG level with HUA risk was also significant in males [OR $(95 \%$ CI $)=2.01 \quad(1.62-2.50) ; \quad p=1.75 \times 10^{-10}$ and OR $(95 \%$

Table 5 Association between ABCG2 rs2231142 variants and HUA according to cardiovascular risk exposure

\begin{tabular}{|c|c|c|c|c|c|c|}
\hline \multirow[t]{2}{*}{ Cardiovascular risk } & \multicolumn{2}{|l|}{ Total } & \multicolumn{2}{|l|}{ Male } & \multicolumn{2}{|l|}{ Female } \\
\hline & $\mathrm{OR}^{\mathrm{a}} 95 \% \mathrm{Cl}$ & $p^{\mathrm{b}}$ & $\mathrm{OR}^{\mathrm{a}} 95 \% \mathrm{Cl}$ & $p^{\mathrm{b}}$ & $\mathrm{OR}^{\mathrm{a}} 95 \% \mathrm{Cl}$ & $p^{\mathrm{b}}$ \\
\hline \multicolumn{7}{|l|}{ Obesity status } \\
\hline $\mathrm{BMl}<24 \mathrm{~kg} / \mathrm{m}^{2}$ & $1.56(1.07-2.31)$ & 0.022 & $1.43(0.84-2.45)$ & 0.191 & $1.51(0.92-2.49)$ & 0.161 \\
\hline $\mathrm{BMI} \geq 24 \mathrm{~kg} / \mathrm{m}^{2}$ & $1.66(1.18-2.36)$ & 0.004 & $1.86(1.15-3.03)$ & 0.012 & $1.59(1.04-3.04)$ & 0.045 \\
\hline \multicolumn{7}{|l|}{ Hypertension } \\
\hline No & $1.43(1.05-2.04)$ & 0.047 & $1.68(1.02-2.78)$ & 0.043 & $1.40(0.81-2.37)$ & 0.219 \\
\hline Yes & $1.88(1.30-2.71)$ & 0.001 & $1.91(1.13-3.24)$ & 0.015 & $1.83(1.09-3.08)$ & 0.021 \\
\hline \multicolumn{7}{|l|}{ Hyper TG } \\
\hline $\mathrm{TG}<1.7 \mathrm{mmol} / \mathrm{L}$ & $1.53(1.14-2.07)$ & 0.005 & $1.58(1.04-2.83)$ & 0.031 & $1.51(0.98-2.33)$ & 0.060 \\
\hline $\mathrm{TG} \geq 1.7 \mathrm{mmol} / \mathrm{L}$ & $2.52(1.33-4.60)$ & 0.003 & $2.41(1.06-4.53)$ & 0.035 & $2.57(1.10-6.01)$ & 0.030 \\
\hline \multicolumn{7}{|l|}{ Hyper LDL } \\
\hline $\mathrm{LDL}<3.4 \mathrm{mmol} / \mathrm{L}$ & $1.43(1.01-2.02)$ & 0.043 & $1.68(1.08-2.62)$ & 0.021 & $1.18(0.66-2.09)$ & 0.582 \\
\hline $\mathrm{LDL} \geq 3.4 \mathrm{mmol} / \mathrm{L}$ & $1.90(1.39-2.79)$ & 0.001 & $1.75(1.12-4.80)$ & 0.024 & $1.98(1.20-3.34)$ & 0.008 \\
\hline \multicolumn{7}{|l|}{ Low HDL } \\
\hline $\mathrm{HDL} \geq 1.0 \mathrm{mmol} / \mathrm{L}$ & $1.57(1.18-2.09)$ & 0.002 & $1.58(1.06-2.34)$ & 0.024 & $1.61(0.93-2.43)$ & 0.087 \\
\hline $\mathrm{HDL}<1.0 \mathrm{mmol} / \mathrm{L}$ & $2.21(1.21-4.01)$ & 0.010 & $2.50(1.12-5.67)$ & 0.026 & $1.92(0.78-4.74)$ & 0.156 \\
\hline
\end{tabular}

a increased odds of HUA associated with each copy of the T allele in ABCG2 rs2231142

${ }^{b} p$ value for the logistic regression adjusted by age, gender, serum cretinine, BMI, hypertension, TG, LDL, and HDL 
Table 6 Association between cardiovascular risk and sUA concentration and HUA risk among ABCG2 T allele carriers

\begin{tabular}{|c|c|c|c|c|}
\hline \multirow{2}{*}{$\begin{array}{l}\text { Cardiovascular } \\
\text { risk factors }\end{array}$} & \multicolumn{2}{|l|}{$s \cup A$} & \multicolumn{2}{|l|}{ hyperuricemia } \\
\hline & Beta (SE) & $p^{*}$ & OR 95\%Cl & $p^{*}$ \\
\hline \multicolumn{5}{|l|}{ Total population } \\
\hline BMI & $0.21(0.60)$ & $3.40 \times 10^{-8}$ & $1.42(1.28-1.56)$ & $4.25 \times 10^{-12}$ \\
\hline Hypertension & $0.036(6.01)$ & 0.978 & $1.35(0.85-2.13)$ & 0.206 \\
\hline TG & $0.24(3.67)$ & $6.18 \times 10^{-9}$ & $1.90(1.44-2.50)$ & $4.08 \times 10^{-6}$ \\
\hline LDL & $0.025(3.77)$ & 0.506 & $1.28(0.94-1.74)$ & 0.114 \\
\hline $\mathrm{HDL}$ & $-0.042(10.76)$ & 0.311 & $0.69(0.25-1.90)$ & 0.471 \\
\hline \multicolumn{5}{|l|}{ Male } \\
\hline BMI & $0.23(1.27)$ & $1.14 \times 10^{-5}$ & $2.01(1.62-2.50)$ & $1.75 \times 10^{-10}$ \\
\hline Hypertension & $0.022(7.83)$ & 0.657 & $1.37(0.74-2.54)$ & 0.319 \\
\hline TG & $0.25(4.72)$ & $8.95 \times 10^{-6}$ & $1.88(1.28-2.74)$ & 0.001 \\
\hline LDL & $0.015(5.27)$ & 0.761 & $1.40(0.84-2.32)$ & 0.192 \\
\hline $\mathrm{HDL}$ & $-0.063(14.92)$ & 0.264 & $0.52(0.11-2.41)$ & 0.407 \\
\hline \multicolumn{5}{|l|}{ Female } \\
\hline BMl & $0.20(1.48)$ & 0.002 & $1.22(1.08-1.37)$ & 0.001 \\
\hline Hypertension & $0.054(9.45)$ & 0.370 & $1.27(0.63-2.55)$ & 0.498 \\
\hline TG & $0.24(5.93)$ & $4.00 \times 10^{-4}$ & $1.95(1.26-3.03)$ & 0.003 \\
\hline LDL & $0.073(5.44)$ & 0.231 & $1.30(0.87-1.41)$ & 0.208 \\
\hline $\mathrm{HDL}$ & $0.025(15.72)$ & 0.711 & $0.83(0.19-3.70)$ & 0.798 \\
\hline
\end{tabular}

${ }^{*} p$ value for the logistic regression adjusted by age, gender, serum cretinine, BMI, hypertension, TG, LDL, and HDL

$\mathrm{CI})=1.88(1.28-2.74) ; p=0.001$, respectively $]$ and females [OR (95\% CI): $1.22(1.08-1.37) ; p=0.001$ and OR (95\% CI): 1.95 (1.26-2.55); $p=0.003$, respectively]. However, other cardiovascular risk factors were not associated with sUA level and HUA risk among rs2231142 T allele carriers.

\section{Discussion}

In the current study, we confirmed that $A B C G 2$ rs2231142 is the most important sUA genetic variant compared to two other sUA genetic polymorphisms, SLC2A9 rs3733591 and $S L C 22 A 12$ rs893006, in the Chinese population. Our data also showed that the combination of the $A B C G 2$ rs2231142 risk allele with two other sUA genetic variants was significantly associated with an increase in sUA level in an elderly Chinese population. Our data are consistent with findings reported by Torres et al. [16], although the studies included different SNPs for SLC2A9 and SLC22A12. In a single-locus analysis, the SLC22A12 SNP rs893006 was reported to be associated with HUA in Japanese men [13]. This locus was related to sUA levels only in males, but there was no significant association with HUA in males or females in the present study, which was in accordance with the findings of a previous study [15]. SNP rs3733591 in SLC2A9 was also identified as being associated with sUA levels and gout in the Han Chinese population [14], but the association was only significant in females in our study. However, the combined effect of the alleles on sUA level was enhanced for carriers of these genetic variants, which indicates that three urate transporter genes interact in the cortical cells in renal tubules. Although our study should be validated with a larger cohort, our findings revealed that assessing the combined effect of genetic factors is more valuable for developing a treatment strategy based on interindividual differences, rather than merely testing for single genotype variants to predict HUA or gout risk.

Genetic factors play an important role in HUA risk, but these factors do not change in a given individual. Thus, our results further demonstrated that cardiovascular risk factors, such as obesity, hypertension, and dyslipidemia, combined with the $A B C G 2$ rs2231142 risk allele, greatly modified the risk of HUA and increased sUA levels. Most importantly, we revealed that $A B C G 2$ rs2231142 T allele carriers with high TG levels have a significantly increased risk of HUA and sUA levels. Associations with sUA levels and lipid metabolism were not clearly reported in a previous study [17], but Fu et al reported that TG, LDL, and HDL levels were significantly associated with HUA risk $(\mathrm{OR}=1.17,1.20$, and 0.62 , respectively) in a Chinese community-dwelling population [2]. Another Chinese prospective study suggested that TG level was a significant and independent risk factor for HUA [4]. The associations found in our study were greatly higher than those reported in previous Chinese studies, possibly due to the older population included in our study. Our findings suggest that more 
attention should be given to controlling serum TG levels and that appropriate diet recommendations or lipidlowering therapy should be provided to elderly Chinese patients, given the high frequency of the $A B C G 2$ rs2231142 risk allele among Asians.

The association of hypertension with HUA risk has been inconsistently reported in the literature $[2,8]$. In our current study, HUA risk was associated with hypertension, but not with DBP or SBP measured at office visits, which could be mainly due to $\mathrm{BP}$ measurements that were possibly affected by anti-hypertension medicine. Furthermore, the proportion of diuretic intake in this the cohort of participations was low and there was no difference between HUA cases and controls; thus, the influence on sUA should be relatively small. Our study also established that HUA risk was increased in ABCG2 rs2231142 risk allele carriers in the hypertensive patients compared to non-hypertension patients. A previously published rodent model demonstrated that hypertension could induce renal arteriosclerosis and tubulointerstitial disease, leading to reduced uric acid excretion and HUA [18]. Therefore, the molecular mechanisms underlying the association remain to be determined.

A previous study using a mouse model showed that $A B C G 2$ expression increased significantly, suggesting a link between enhanced urate reabsorption and obesityassociated HUA [19]. Our current data further confirmed that both obese males and females carrying the rs2231142 $\mathrm{T}$ allele has an associated increased HUA risk, which was consistent with other Asian population studies $[8,20]$. However, a previous study in a European population only showed a significant association in males, not in females [21], possibly due to ethnic differences. Estrogen levels can affect $A B C G 2$ expression [22, 23 ], thus we excluded postmenopausal women to eliminate the effect of estrogen on allelic associations with HUA.

Our current study does have some limitations. For example, we did not include all cardiovascular risk factors that could impact sUA levels and HUA risk, such as lifestyle and dietary factors. In addition, more samples from different districts need to be included in future analyses to better investigate the association of $A B C G 2$ polymorphisms and other genetic variants and cardiovascular risk factors with the risk of developing HUA.

\section{Conclusions}

Our current study demonstrated that the $A B C G 2$ rs2231142 variant in combination with the SLC2A9 and SLC22A12 genetic variants significantly increased sUA levels in a Chinese community population. The $A B C G 2$ rs2231142 variant in combination with cardiovascular risk factors, specifically higher TG levels, is significantly associated with increased sUA levels and HUA risk.

\section{Abbreviations}

ABCG2: ATP-binding cassette subfamily G member 2; BMI: Body mass index; Ccr: Creatinine clearance; DBP: Diastolic blood pressure; FBG: Fasting bloodglucose; GLUT9: Glucose transporter type 9; GWAS: Genome-wide association studies; HDL: High density lipoprotein; LDL: Low-density lipoprotein; MAF: Minor allele frequencies; SBP: Systolic blood pressure; SD: Standard deviation; SNP: Single nucleotide polymorphism; sUA: serum uric acid; TG: Triglyceridemia; URAT1: Urate anion transporter 1

\section{Acknowledgements}

The authors would like to thank all study individuals for their participation in this study.

\section{Authors' contributions}

$J L$ and $W Y$ conceived and designed the experiments; $J L$ prepared the manuscript; $W Y$ and $Y L$ reviewed manuscript; $J$, $X D$, and $Z W$ performed clinic interviews and laboratory experiments, as well as data analysis. All authors read and approved the final manuscript.

\section{Funding}

This study was supported in part by grants from the National Natural Science Foundation of China (\#81601109) and the Beijing Municipal Excellent Talents Foundation (\#2016000020124G107).

\section{Availability of data and materials}

According to the Chinese policy of "National regulation on the management of human genetic resources" released by State Council (Index No. 000014349/2019-00063; Serial No. 171), availability of the data and materials is confidential.

\section{Ethics approval and consent to participate}

This study was approved by the Ethics Committee of Xuan Wu Hospital (Beijing, China), and all participants signed informed consent before enrolling in this study.

Consent for publication

Not Applicable.

\section{Competing interests}

The authors declare that they have no competing interests.

\section{Author details}

${ }^{1}$ Departments of Geriatric Medicine, Xuanwu Hospital, The Capital Medical University, 45 Changchun Street, Xicheng District, Beijing 100053, China. ${ }^{2}$ Departments of Neurology, Xuanwu Hospital, The Capital Medical University, Beijing, China.

Received: 29 April 2019 Accepted: 28 February 2020

Published online: 17 March 2020

\section{References}

1. Zhu Y, Pandya BJ, Choi HK. Prevalence of gout and hyperuricemia in the US general population: the National Health and nutrition examination survey 2007-2008. Arthritis Rheum. 2011;63:3136-41.

2. Fu S, Luo L, Ye P, Xiao W. Epidemiological associations between hyperuricemia and cardiometabolic risk factors: a comprehensive study from Chinese community. BMC Cardiovasc Disord. 2015;15:129.

3. Lu X, Li X, Zhao Y, Zheng Z, Guan S, Chan P. Contemporary epidemiology of gout and hyperuricemia in community elderly in Beijing. Int J Rheum Dis. 2014;17:400-7.

4. Zhang Y, Wei F, Chen C, Cai C, Zhang K, Sun N, et al. Higher triglyceride level predicts hyperuricemia: a prospective study of 6-year follow-up. J Clin Lipidol. 2018;12:185-92.

5. Dehghan A, Kottgen A, Yang Q, Hwang SJ, Kao WL, Rivadeneira F, et al. Association of three genetic loci with uric acid concentration and risk of gout: a genome-wide association study. Lancet. 2008;372:1953-61.

6. Kottgen A, Albrecht E, Teumer A, Vitart V, Krumsiek J, Hundertmark C, et al. Genome-wide association analyses identify 18 new loci associated with serum urate concentrations. Nat Genet. 2013;45:145-54. 
7. Woodward OM, Kottgen A, Coresh J, Boerwinkle E, Guggino WB, Kottgen M Identification of a urate transporter, $A B C G 2$, with a common functional polymorphism causing gout. Proc Natl Acad Sci U S A. 2009;106:10338-42.

8. Takeuchi F, Yamamoto K, Isono M, Katsuya T, Akiyama K, Ohnaka K, et al. Genetic impact on uric acid concentration and hyperuricemia in the Japanese population. J Atheroscler Thromb. 2013;20:351-67.

9. Okada Y, Sim X, Go MJ, Wu JY, Gu D, Takeuchi F, et al. Meta-analysis identifies multiple loci associated with kidney function-related traits in east Asian populations. Nat Genet. 2012;44:904-9.

10. Cao J, Zhang J, Li Q, Jiang C, Song Y, Liu C, et al. Serum phosphate and the risk of new-onset hyperuricemia in hypertensive patients. Hypertension. 2019;74(1):102-10.

11. Williams B, Mancia G, Spiering W, Agabiti Rosei E, Azizi M, Burnier M, et al. 2018 ESH/ESC guidelines for the management of arterial hypertension. Eur Heart J. 2018:39(33):3021-104.

12. Stone NJ, Robinson JG, Lichtenstein AH, et al. American College of Cardiology/ American Heart Association task force on practice G. 2013 ACC/ AHA guideline on the treatment of blood cholesterol to reduce atherosclerotic cardiovascular risk in adults: a report of the American College of Cardiology/American Heart Association task force on practice guidelines. J Am Coll Cardiol. 2014;63:2889-934.

13. Shima Y, Teruya K, Ohta H. Association between intronic SNP in urate-anion exchanger gene, SLC22A12, and serum uric acid levels in Japanese. Life Sci. 2006;79:2234-7.

14. Tu HP, Chen CJ, Tovosia S, Ko AM, Lee CH, Ou TT, et al. Associations of a non-synonymous variant in SLC2A9 with gouty arthritis and uric acid levels in Han Chinese subjects and Solomon islanders. Ann Rheum Dis. 2010;69: 887-90.

15. Yang X, Xiao Y, Liu K, et al. Prevalence of hyperuciemia among the Chinese population of the southeast coastal region and association with single nucleotide polymorphisms in urate-anion exchanger genes: SLC22A12, ABCG2 and SLC2A9. Mol Med Rep. 2018;18(3):3050-8.

16. Torres RJ, de Miquel E, Bailén, Banegas JR, Puig JG. Tubular Urate transporter gene polymorphisms differentiate patients with gout who have normal and decreased urinary uric acid excretion. J Rheumatol. 2014;41(9):1863-70.

17. Lippi G, Montagnana M, Luca Salvagno G, Targher G, Cesare GG. Epidemiological association between uric acid concentration in plasma, lipoprotein(a), and the traditional lipid profile. Clin Cardiol. 2010;33:E76-80.

18. Wang J, Qin T, Chen J, Li Y, Wang L, Huang H, et al. Hyperuricemia and risk of incident hypertension: a systematic review and meta-analysis of observational studies. PLoS One. 2014;9:e114259.

19. Doshi M, Takiue Y, Saito H, Hosoyamada M. The increased protein level of URAT1 was observed in obesity/metabolic syndrome model mice. Nucleosides Nucleotides Nucleic Acids. 2011;30:1290-4.

20. Cheng ST, Wu S, Su CW, Teng MS, Hsu LA, Ko YL. Association of ABCG2 rs2231142-a allele and serum uric acid levels in male and obese individuals in a Han Taiwanese population. J Formos Med Assoc. 2017;116:18-23.

21. Huffman JE, Albrecht E, Teumer A, Mangino M, Kapur K, Johnson T, et al. Modulation of genetic associations with serum urate levels by body-massindex in humans. PLoS One. 2015;10:e0119752.

22. Ee PL, Kamalakaran S, Tonetti D, He X, Ross DD, Beck WT. Identification of a novel estrogen response element in the breast cancer resistance protein (ABCG2) gene. Cancer Res. 2004;64:1247-51.

23. Chang FW, Fan HC, Liu JM, Fan TP, Jing J, Yang CL, et al. Estrogen enhances the expression of the multidrug transporter gene ABCG2-increasing drug resistance of breast Cancer cells through estrogen receptors. Int J Mol Sci. 2017;18:163.

\section{Publisher's Note}

Springer Nature remains neutral with regard to jurisdictional claims in published maps and institutional affiliations.

Ready to submit your research? Choose BMC and benefit from:

- fast, convenient online submission

- thorough peer review by experienced researchers in your field

- rapid publication on acceptance

- support for research data, including large and complex data types

- gold Open Access which fosters wider collaboration and increased citations

- maximum visibility for your research: over $100 \mathrm{M}$ website views per year

At BMC, research is always in progress.

Learn more biomedcentral.com/submissions 